\title{
Removal and effects of scatter-glare in cone-beam CT with an amorphous-silicon flat-panel detector
}

\author{
G Poludniowski, P M Evans, A Kavanagh and S Webb \\ Joint Department of Physics, \\ Institute of Cancer Research and Royal Marsden NHS Foundation Trust, \\ Downs Road, Sutton, Surrey, SM2 5PT UK \\ E-mail: Gavin.Poludniowski@icr.ac.uk
}

\begin{abstract}
.
Scatter in a detector and its housing can result in image degradation. Typically, such scatter leads to a low-spatial frequency 'glare' superimposed on the primary signal. We infer the glare-spread-function (GSF) of an amorphous-silicon flat-panel detector via an edge-spread technique. We demonstrate that this spread (referred to as 'scatter-glare' herein) causes a low-spatial-frequency drop in the associated modulationtransfer-function. This results in a compression of the range of reconstructed CTnumbers and is an impediment to accurate CT-number calibration. We show that it can also lead to visual artefacts. This explains previously unresolved CT-number discrepancies in an earlier work (Poludniowski et al 2009a Phys. Med. Biol. 54 3847). We demonstrate that after deconvolving the GSF from the projection images, in conjunction with a correction for phantom-scatter, the CT-number discrepancies disappear. We show results for an in-house-built phantom with inserts of tissueequivalent materials and for a patient scan. We conclude that where scatter-glare has not been accounted for the calibration of cone-beam CT-numbers to material density will be compromised. The scatter-glare measurement method we propose is simple and requires no special equipment. The deconvolution process is also straight-forward and relatively quick (60 ms per projection on a desktop PC).
\end{abstract}

\section{Introduction}

In recent years treatment-room cone-beam computed tomography (CBCT) has become a reality, thanks to the introduction of gantry-mounted x-ray tubes and flat-panel detectors on clinical linacs. In current clinical usage, such CBCT devices are typically used for position-verification and correcting patient shifts (see e.g. Den et al 2010). It is widely recognized that their potential is far greater than this (e.g. Richter et al 2008 and Boggula et al 2009). If accurate CT numbers could be produced, CBCT scans could be used for dose-verification and potentially adaptive radiotherapy, as described by Lu et al (2008) and Webb (2008). Body (patient or phantom) scatter is a well-known cause of artefacts and CT-number inaccuracy in CBCT (Siewerdsen and Jaffray 2001). There have been diverse approaches to solving the problem of scatter. These have ranged from 
a detailed and computationally-demanding Monte Carlo approach (Jarry et al 2006) to entirely empirical measures (Marchant et al 2008). We have previously described an accelerated Monte Carlo-based approach (Poludniowski et al 2009a). The method was fast and corrected the background CT numbers effectively. However, we observed a residual problem: there was a compression of the CT-number range. Regions of high density (dense bone) were of lower CT-number than expected while those of low density (air) were higher.

In this paper we estimate the line-spread-function (LSF) and hence infer a pointspread-function (PSF) of an amorphous-silicon (a-Si) flat-panel detector system using an edge-spread technique. Such edge-spread techniques have been widely used to determine both the modulation transfer function (MTF) of imaging systems (e.g. Illers et al 2005) and to derive scatter kernels (e.g. Li et al 2008). We find that the tails of the PSF result in low-spatial-frequency drop in the associated modulation-transfer-function (MTF). This phenomenon will be referred to as scatter-glare. Scatter-glare is analogous to veiling-glare in image-intensifier devices, but is not primarily caused by the diffusion of optical photons in a scintillator. Rather, it is assumed to be largely generated by the scattering of incident x-ray photons in the detector panel and its housing. Glaring (and hence a low-spatial-frequency drop) is a well-known effect in image-intensifier devices (Seibert et al 1984) but has also been observed in flat-panel detectors (e.g. Cooper III et al 2000, Friedman and Cunningham 2008, Carton et al 2009). We demonstrate that this phenomenon explains the above-noted discrepancy in our previous work (Poludniowski et al 2009a). The tails of the PSF are parameterized herein by a glare-spread-function (GSF). After deconvolving the GSF from the projection images, the CT-number accuracy of the CBCT reconstructions is dramatically improved. An in-house-built phantom with inserts of tissue-equivalent materials was used for this purpose. We conclude that correcting for body-scatter in CBCT is not enough for quantitative purposes: scatter within the panel and housing must also be accounted for. Deconvolution of this component allows the accurate cross-calibration of a CBCT unit to a planning CT scanner.

\section{Theory and methods}

Synergy XVI CBCT units (Elekta, Crawley, UK) were used for this study. These units consist of a gantry-mounted x-ray tube and flat-panel detector. The flat-panel is based on a-Si technology coupled with a columnar $\mathrm{CsI}(\mathrm{Tl})$ scintillator and has an active area of $41 \times 41 \mathrm{~cm}^{2}$. Image files were generated as $512 \times 512$ pixel images in HIS format (PerkinElmer Optoelectronics, Fremont CA, USA). An edge-spread distribution was measured for the panel, using an experimental setup as illustrated in figure 1 (a) and (b). Half the area of the panel was shielded by lead sheeting of a total thickness in excess of $6 \mathrm{~mm}$. The edge of the sheeting was aligned using treatment-room laser-positioning. The lead was placed on the patient support system at approximately $30 \mathrm{~cm}$ from the imaging plane. The source-to-detector distance was nominally $153.6 \mathrm{~cm}$. The lead 
collimation was performed relatively close to the panel to minimize blurring effects from the finite focal spot of the x-ray tube. The resulting de-magnification (magnification: about 0.25 ) puts focal-spot blurring at the sub-pixel level (Chen et al 2008). Projection o images were acquired at $80 \mathrm{kV}$ (1.024 mAs/projection), $100 \mathrm{kV}$ (0.625 mAs/projection) or $120 \mathrm{kV}$ (0.320 mAs/projection). Images were also acquired with two different XVI units (designated Unit 1 and Unit 2). Normalization (full-field) exposures were acquired to correct for pixel-to-pixel variations in gain. In all cases, care was taken to ensure that the digital value (DV) of the detector did not saturate. An LSF can be found from the derivative of the edge-spread distribution (Barrett and Swindell 1981). For pixels of width, $\Delta x$, the experimental LSF at the $i$ th pixel can be approximated as,

$$
L S F^{(\exp )}\left(x_{i}\right) \propto \frac{D V_{i-1}-D V_{i+1}}{2 \Delta x}
$$

where $x_{i}$ is the centre of the $i$ th pixel where $i$ is incremented in the direction of the edge-transition (the orthogonal coordinate being $y_{j}$ ). One-hundred LSF line-profiles were averaged to calculate $L S F^{(\exp )}$ (to reduce the effects of noise). A Matlab script (MathWorks Inc., Natwick MA, USA) was written to minimize a cost-function for agreement between a LSF fitting function $\left(L S F^{(f i t)}\right)$ and the experimental estimate $\left(L S F^{(e x p)}\right)$. The cost-function, $X^{2}$, was chosen as a weighted-least-squares:

$$
X^{2}=\sum_{i} W_{i}\left(L S F^{(e x p)}\left(x_{i}\right)-L S F^{(f i t)}\left(x_{i}\right)\right)^{2},
$$

where

$$
W_{i}=\frac{x_{i}^{2}}{\sigma^{2}\left(x_{i}\right)} .
$$

In equation (3) $\sigma\left(x_{i}\right)$ is the standard deviation in $\operatorname{LSF}^{(e x p)}\left(x_{i}\right)$ derived from the set distribution and de-emphasizes noisy data. The fitting function was chosen as,

$$
L S F^{(f i t)}(x)=\frac{a_{1}}{\sqrt{2 \pi b_{1}^{2}}} e^{-|x|^{2} / 2 b_{1}^{2}}+\frac{a_{2}}{\pi b_{2}^{2}}|x| K_{1}\left(\frac{|x|}{b_{2}}\right)+\frac{a_{3}}{\pi b_{3}} \frac{1}{1+|x|^{2} / b_{3}^{2}}
$$

where $a_{i}$ and $b_{i}$ are fitting coefficients and $K_{1}(\ldots)$ is a 1st-order MacDonald (modified Bessel) function (Lebedev 1972). Due to the normalization constraint that $a_{1}+a_{2}+a_{3}=$ 1 there are 5 fitting parameters. Expression (4) is inelegant but has some advantages as a fitting function. Firstly, the three constituent functions (Gaussian, MacDonald and Lorentzian) have varying degrees of kurtosis. This means that the combined function can describe a large family of distributions. Secondly, the calculations of the PSF and MTF from this LSF are analytically tractable and the results can be expressed without resort to special functions. For the purposes of this work we assume that:

- The PSF of the detector is stationary (does not vary across the panel);

- This PSF is circularly symmetric (i.e. dependent only on $|r|=\sqrt{x^{2}+y^{2}}$ ).

The first assumption is essential for any deconvolution using standard Fourier transform techniques. There is no reason to suggest that the latter assumption is invalid (except 
for any residual focal-spot blurring contribution). The above form for the LSF fitting

function implies a PSF:

$$
P S F^{(f i t)}(r)=\frac{a_{1}}{2 \pi b_{1}^{2}} e^{-r^{2} / 2 b_{1}^{2}}+\frac{a_{2}}{2 \pi b_{2}^{2}} e^{-r / b_{2}}+\frac{a_{3}}{2 \pi b_{3}^{2}} \frac{1}{\left(1+r^{2} / b_{3}^{2}\right)^{3 / 2}}
$$

where the fitting coefficients take the same numerical values as in (4). This can be shown using identities (A1-A3) of Appendix A and the relation

$$
L S F^{(f i t)}(x)=\int_{-\infty}^{\infty} P S F^{(f i t)}(r(x, y)) d y .
$$

It is tempting to attribute the three terms in (5) as modelling the distributions of the dose deposition in the scintillator, the diffusion of scattered optical photons, and, the scatter of x-ray photons in the detector panel and housing, respectively. We will, however, treat equation (5) as an empirical model for the LSF, that is: the parameters $\left\{a_{2}, a_{3}, b_{1}, b_{2}, b_{3}\right\}$ are varied freely as fitting parameters. We note that an exponential form for the PSF due to the diffusion of optical photons in a thin scintillator has been derived in the literature by Seibert et al 1984. The first two terms of (5) correspond to their model, except that in their work they used a Gaussian with zero width (i.e. a Dirac delta-function). We observe that with a different scintillator and within a mammography context Cooper III et al (2000) were able to fit the tails of a flat-panel detector PSF adequately without a third non-exponential term. A third term was needed in this work to model the long-range glare. We limit our physical interpretation of (4) to the statement that the presence of the Lorentzian function is largely due to x-ray scatter (see Appendix B). Also note that the $b_{1}$ parameter of the Gaussian in particular is likely to depend on the experimental technique (see Discussion). The MTF corresponding to (5) (i.e. the normalized modulus of the 2D Fourier Transform of the PSF) takes the form,

$$
\operatorname{MTF}^{(f i t)}(\omega)=a_{1} e^{-b_{1}^{2} \omega^{2} / 2}+a_{2} \frac{1}{\left(1+b_{2}^{2} \omega^{2}\right)^{3 / 2}}+a_{3} e^{-b_{3} \omega},
$$

where $\omega$ is the spatial (angular) frequency. This can be shown using identities (A4-A7) of Appendix A. The fitting coefficients, derived from the LSF, were used to deconvolve the glare from experimental projections. A program was written in Fortran 95 to do this in the frequency domain using Fast Fourier Transforms (FFTs). The 2D FFT facility of the Intel Math Kernel, supplied with Intel Visual Fortran compiler v11.0 (Intel Corporation, Santa Clara CA, USA), was used. The PSF itself was not used for deconvolution, as complete deblurring was unstable in the high-spatial-frequency region. Instead, a glare-spread-function was used as the basis of the deconvolution kernel. We define this to be:

$$
\begin{aligned}
G S F(r) & =\lim _{b_{1}, b_{2} \rightarrow 0} P S F(r) \\
& =\left(1-a_{3}\right) \frac{\delta(r)}{2 \pi r}+\frac{a_{3}}{2 \pi b_{3}^{2}} \frac{1}{\left(1+r^{2} / b_{3}^{2}\right)^{\frac{3}{2}}},
\end{aligned}
$$

where the non-exponential portion of the PSF models the long-range tails of the distribution. A theoretical justification for the form of (9) is presented in Appendix 
B. A discrete approximation of the GSF can then be defined as,

$$
G S F_{i, j}=\left(1-a_{3}\right) \delta_{i, 0} \delta_{j, 0}+\frac{a_{3} \Delta x \Delta y}{2 \pi b_{3}^{2}} \frac{1}{\left(1+r_{i, j}^{2} / b_{3}^{2}\right)^{\frac{3}{2}}},
$$

where the function of a Dirac delta $(\delta(r) / 2 \pi r)$ is replaced by Kronecker deltas $\left(\delta_{i, 0}\right.$ etc.), $\Delta x \Delta y$ is the area of a pixel and $r_{i, j}=\sqrt{x_{i}^{2}+y_{j}^{2}}$. The deconvolution operation can then be summarized by the expression,

$$
D V_{i, j}^{(c o r r)}=F F T^{-1}\left\{\frac{F F T\left\{D V_{i, j}\right\}}{F F T\left\{G S F_{i, j}\right\}}\right\}
$$

where $D V^{(\text {corr })}$ is the glare-corrected image and each 2D FFT (or inverse: $\mathrm{FFT}^{-1}$ ) is appropriately zero-padded.

A phantom was built for the purpose of calibrating CT-numbers in CBCT. The Hounsfield Unit calibration phantom (HOUNDphan) is shown in figure 2 (a). Removable inserts of several tissue-equivalent materials (in addition to water and air) were available: LN10 (lung), AP7 (adipose), WT1 (wet-tissue), IB7 (internal bone), RB2 (rib-bone) and SB5 (cortical-bone). These materials were supplied by the Tissue Substitute Section of St Bartholomew's Hospital (London, UK) and the compositions and densities are presented in table $1 \ddagger$. The body of the phantom was manufactured from WT1. A Synergy XVI reconstruction of HOUNDphan produced by Elekta's commercial software is shown in figure 2 (b) (full-scan acquisition, $100 \mathrm{kV}, 0.625 \mathrm{mAs} /$ projection, F0 cassette) The default clinical scatter correction was used. The insert materials correspond to those superimposed on figure 2 (a). An 'S2' cassette was used for source collimation. This was manufactured in-house by the adaptation of an existing cassette. It is equivalent to the standard Elekta M2 cassette, but made for the smallest of the three available fields-of-view. We note that the CT-numbers produced by the Elekta software are not (nor are intended to be) quantitatively accurate. However, observe the bright and dark artefacts emanating from the material inserts in the reconstruction of figure 2 (b). The correction of these artefacts will be addressed in this work. We further note that there is a narrow region of too low CT-number present in the bottom right-hand corner of the image (indicated in the figure). This artefact is not due to body-scatter or scatter-glare, but rather exposure-dependent variations in pixel signal gain (ghosting) (Siewerdsen and Jaffray 1999). The artefact is particularly apparent for the HOUNDphan phantom because of its close to, but not completely, circular cross-section. It could, potentially, be removed by established methods (Mail et al 2008, Ploeger et al 2010).

The same CBCT projections that were used to generate figure 2 (b) were subsequently used in reconstructions with an in-house implementation of the Feldkamp algorithm (Feldkamp et al 1984, Kak and Slaney 2001). Reconstructions were performed with and without scatter-glare removal and with and without removal of body-scatter. The body-scatter was estimated using a fast Monte Carlo code described previously

$\ddagger$ Compositions were derived from information provided by the Tissue Substitute Section of St Bartholomew's Hospital (London, UK). Density estimations were based on measurements conducted in our department on the tissue-equivalent samples. 
Table 1. Percentage composition (by weight) and density of tissue-equivalent materials.

\begin{tabular}{|c|ccccccc|c|}
\hline Material & $\mathrm{H}$ & $\mathrm{C}$ & $\mathrm{N}$ & $\mathrm{O}$ & $\mathrm{Cl}$ & $\mathrm{Ca}$ & $\mathrm{F}$ & $\rho\left[\mathrm{g} / \mathrm{cm}^{3}\right]$ \\
\hline LN10 & 8.4 & 68.0 & 2.3 & 18.9 & 0.1 & 2.4 & 0.0 & 0.27 \\
AP7 & 8.4 & 69.1 & 2.4 & 16.9 & 0.1 & 0.0 & 3.1 & 0.95 \\
WT1 & 8.4 & 68.0 & 2.3 & 18.9 & 0.1 & 2.4 & 0.0 & 1.00 \\
IB7 & 7.1 & 59.6 & 2.0 & 23.4 & 0.1 & 7.8 & 0.0 & 1.10 \\
RB2 & 5.7 & 50.6 & 1.7 & 28.2 & 0.1 & 13.7 & 0.0 & 1.29 \\
SB5 & 2.6 & 30.6 & 1.0 & 38.9 & 0.1 & 26.9 & 0.0 & 1.84 \\
\hline
\end{tabular}

175 (Poludniowski et al 2009a). The energy response of the flat-panel detector was taken from the work of Roberts et al (2008). The x-ray spectrum of the x-ray tube was calculated for using the program SpekCalc (Poludniowski et al 2009b) based on a published model (Poludniowski 2007) and known half-value-layer (HVL) data. A diagnostic-quality CT scan of the HOUNDphan phantom was obtained using a planning CT scanner (Brilliance CT Big Bore scanner, Philips, Best, NL) in axial mode $(120 \mathrm{kV}$, $1.5 \mathrm{~mm}$ slice thickness). This was used as the basis of the Monte Carlo calculations, using the known densities of the constituent materials and their correspondence with CT-number. The detector signal due to body-scatter was calculated for a grid of 32x32 node points at the flat-panel at 90 equally spaced angles over $2 \pi$ radians. Each projection consisted of 2000 histories (note that the use of forced detection and quasi-random numbers necessitates fewer histories than in a conventional Monte Carlo simulation). To rigidly register the planning CT scan to the CBCT scan, prior to Monte Carlo calculation, the rreg and transformation tools of the IRTK toolkit were used (Studholme et al 1999). The other computationally intensive parts of the process (Monte Carlo simulation, CBCT reconstruction and glare deconvolution) were coded in Fortran 95. The final CBCT reconstructions were produced in DICOM format. Image conversion and manipulation was handled using scripts written in Python 2.6 (Python Software Foundation). Various publically available software programs and modules were utilized $\S$.

To compare material CT-number accuracy with and without phantom-scatter and scatter-glare removal, it was necessary to calculate expected CT-number for the various inserts. This was done by performing a reconstruction using simulated primary-only projections (again modelling the x-ray spectrum and panel-response). For this purpose, $128 \times 128$ pixel projections through the planning CT scan were calculated at 360 equally spaced angles over $2 \pi$ radians. For all reconstructions, the reconstructed CT-numbers of inserts were found by placing regions-of-interest (approximately 100 pixels) in the central slice and taking the mean value.

$\S$ PyDicom (available from: http://code.google.com/p/pydicom/); dicom2nii.exe (available from: http://www.sph.sc.edu/comd/rorden/MRicron/); IRTK (available from: http://www.doc.ic.ac.uk/ dr/software/) and PyNifti (available from: http://niftilib.sourceforge.net/pynifti/). 
(a)

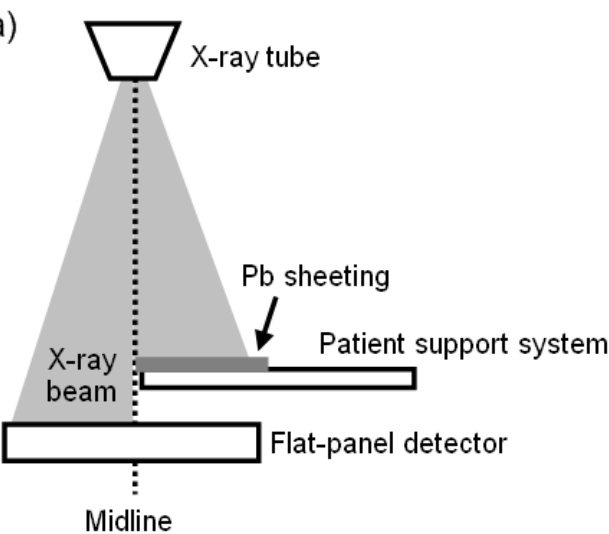

(b)

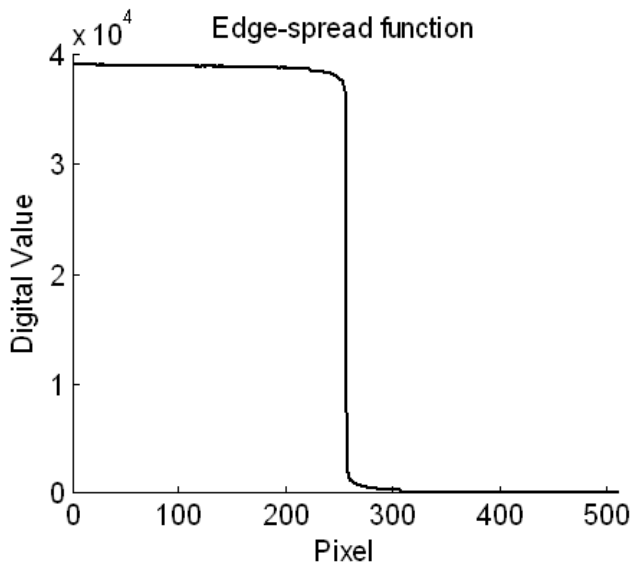

Figure 1. (a) The experimental setup for the edge-spread experiment and (b) a typical example of an averaged edge-spread distribution.

(a)

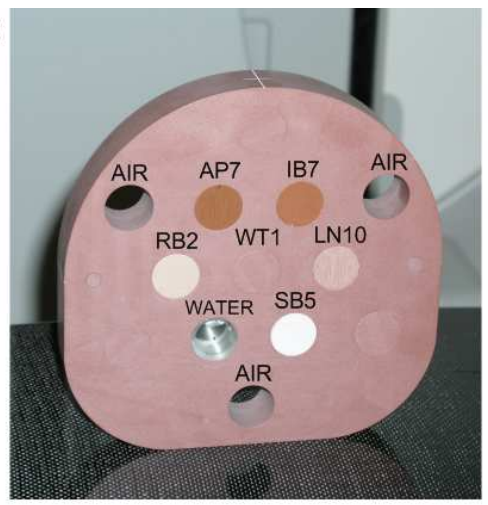

(b)

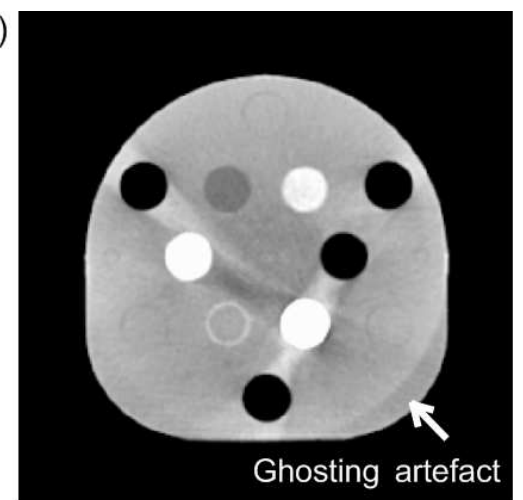

Figure 2. (a) A photograph of the HOUNDphan phantom and (b) an XVI reconstruction of the phantom using Elekta's software.

\section{Results}

The experimental LSF data are shown in figure 3 (a) for: Unit 1 at $80 \mathrm{kV}$ (circles), $100 \mathrm{kV}$ (triangles) and $120 \mathrm{kV}$ (squares), and for Unit 2 at $100 \mathrm{kV}$ (stars). All data sets closely coincide. For that reason a global fit to all data sets was conducted simultaneously. The resulting fitting coefficients are presented in table 2 . We observe that the data points in figure 3 (a) are noisier for negative than positive displacements. This is because these points are in the open-field and therefore subject to greater stochastic fluctuation (although the relative noise in the edge-spread distribution is less in this region). Figure 3 (b) shows the edge-spread estimate of the MTF (solid line) calculated from the fitting coefficients of table 2 and equation (6). Note the sharp drop in the MTF between zero and about 0.25 radians $\cdot \mathrm{mm}^{-1}$. This low-spatial-frequency drop is modelled by the exponential component of (6) (i.e. Lorentzian component of the LSF). The broken-curve shows the GSF-corrected MTF (i.e. the panel MTF divided 
by the 2D Fourier transform of the GSF). The low-spatial-frequency drop has been corrected.
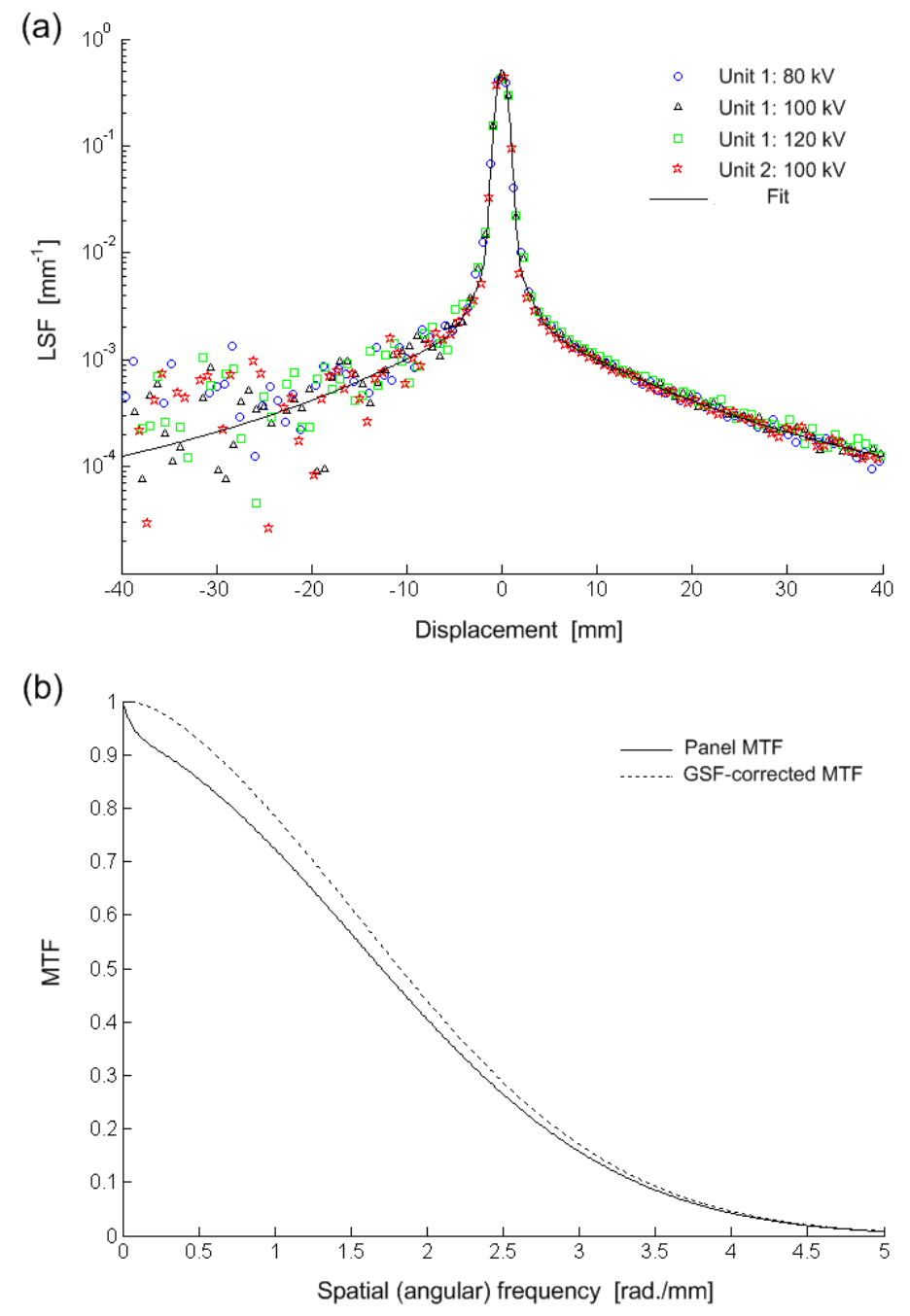

Figure 3. The LSFs for XVI Unit $1(80,100$ and $120 \mathrm{kV})$ and Unit $2(100 \mathrm{kV})$ and the global fit (a); the panel MTF and glare-corrected MTF corresponding to the global fit (b).

A simulated line profile through the central-plane of the HOUNDphan phantom, for the case where the x-ray source is directly above the phantom, is shown in figure 4 . Both the primary and body-scatter components are shown. Despite the narrowness of the phantom and the beam collimation, the scatter component remains non-negligible (albeit small: a maximum scatter-to-primary ratio of 0.68). Body-scatter must therefore be corrected for.

Figure 5 (a) shows the central slice of the planning CT scan of the HOUNDphan phantom, with the visualization window set to -250 to +250 HU. Figure 5 (b), with the same windowing, shows the CBCT reconstruction without body-scatter or scatterglare correction. Marked artefacts very similar to those apparent with the Elekta XVI 
Table 2. Global fitting coefficients for the XVI flat-panel LSF/PSF/GSF.

\begin{tabular}{|c|c|}
\hline Coefficient & Value \\
\hline$a_{1}$ & 0.8563 \\
$a_{2}$ & 0.0650 \\
$a_{3}$ & 0.0787 \\
\hline Coefficient & Value $[\mathrm{mm}]$ \\
\hline$b_{1}$ & 0.4918 \\
$b_{2}$ & 1.0455 \\
$b_{3}$ & 10.6244 \\
\hline
\end{tabular}

software (figure $2(\mathrm{~b})$ ) are apparent. The CT numbers are also, predominately, too low. Figure 5 (c) shows the same reconstruction with body-scatter correction but no removal of scatter-glare. The CT-number values are partially corrected. Artefacts still clearly remain, however. In figure 5 (d), both body-scatter and scatter-glare have been corrected for. The visual artefacts are now almost completely removed.

Figure 6 (a) shows the expected CT-numbers for the various materials (dots) compared with the calculated values associated with the slices shown in figures 5 (b) (dashed line), (c) (dotted lines) and (d) (solid line). The error bars displayed are twice the pixel standard deviation in the relevant region-of-interest. It is clear from the plot that although correcting for body-scatter improves the agreement towards expectation, without correction for the glare, close quantitative agreement remains poor and the CT-number range compressed. Furthermore, comparison of the error bars in figure 6 (a) shows that deconvolution does not have an appreciable detrimental effect on reconstruction noise. This is because it is predominantly the low-spatial-frequencies that are modified by the GSF filter. Figure 6 (b) shows, for the various materials, the CT-numbers in the planning scan plotted against those for the CBCT reconstructions. Such a calibration curve provides a mechanism (e.g. look-up-table) for producing a CBCT scan equivalent (in terms of $\mathrm{HU}$ ) to that produced by a specified planning $\mathrm{CT}$ scanner.

To illustrate the implications of scatter-glare in a clinical scenario, Figure 7 shows an axial planning CT slice reconstructed through the head of a head-and-neck cancer patient. The corresponding slice from a treatment-room CBCT scan is also shown. Four regions-of-interest (ROIs) are depicted. Table 3 presents the average CT number in the ROIs for the planning CT scan and the errors with respect to this for three CBCT reconstructions. All three cone-beam reconstructions used the same projection data and the HU look-up-table presented in figure 6 (b). Different levels of corrections were, however, applied. The trends in the errors are consistent with expectation. Without any correction for body-scatter or scatter-glare errors ranged from -360 to $+180 \mathrm{HU}$ (Case 1). Correction for only body-scatter improved the HU agreement (Case 2), but large discrepancies remained where the expected CT-number was very different 
to the background soft-tissue e.g. in dense bone $(-298)$ or an air cavity $(+121)$. When both body-scatter and scatter-glare corrections were applied (Case 3), the CT number discrepancies improved considerably, ranging from -45 to +42 .

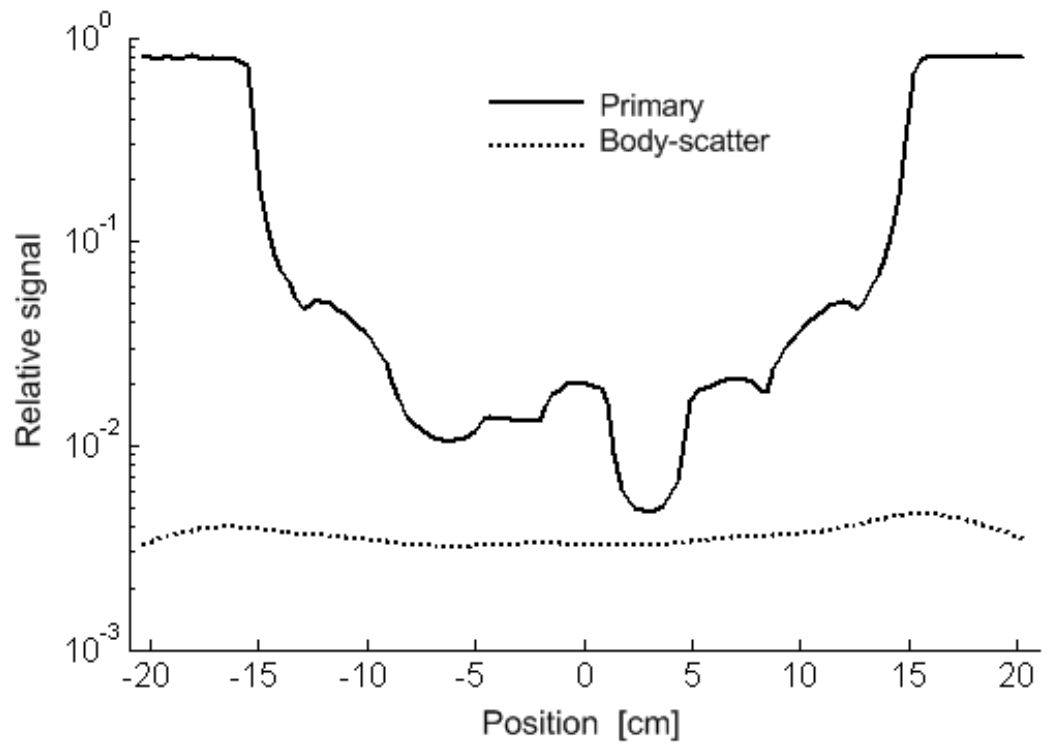

Figure 4. Line-profile of simulated signal through the central-plane of the HOUNDphan phantom. Primary and body-scatter components are shown.

Table 3. Planning CT-numbers and relative CBCT errors for a head-and-neck cancer patient. All values quoted are calculated from the mean CT-number in an ROI, averaged over 10 repeat-draws of the ROI in the same approximate position. Values in round brackets are the corresponding standard errors on the means due to ROI delineation/positioning.

\begin{tabular}{|c|c|ccc|}
\hline & Planning CT & & CBCT Error & {$[\mathrm{HU}]$} \\
ROI & CT-number [HU] & Case 1 & Case 2 & Case 3 \\
\hline 1. Air (external) & $-1001(0)$ & $+24(2)$ & $+6(2)$ & $+4(1)$ \\
2. Air (hypopharynx) & $-981(0)$ & $+180(1)$ & $+121(1)$ & $+42(2)$ \\
3. Soft-tissue & $17(1)$ & $-48(2)$ & $+19(1)$ & $+19(1)$ \\
4. Jaw bone & $851(35)$ & $-360(17)$ & $-298(8)$ & $-45(11)$ \\
\hline
\end{tabular}

\section{Discussion}

A general expression, adequate to fit experimentally observed LSFs, has been proposed in equation (4). We presented fitting coefficients in table 2. These coefficients define an LSF (and hence PSF and MTF) for a generic XVI flat-panel at clinically relevant x-ray tube potentials $(80-120 \mathrm{kV})$. Of course, a panel-specific LSF based on measurements for 

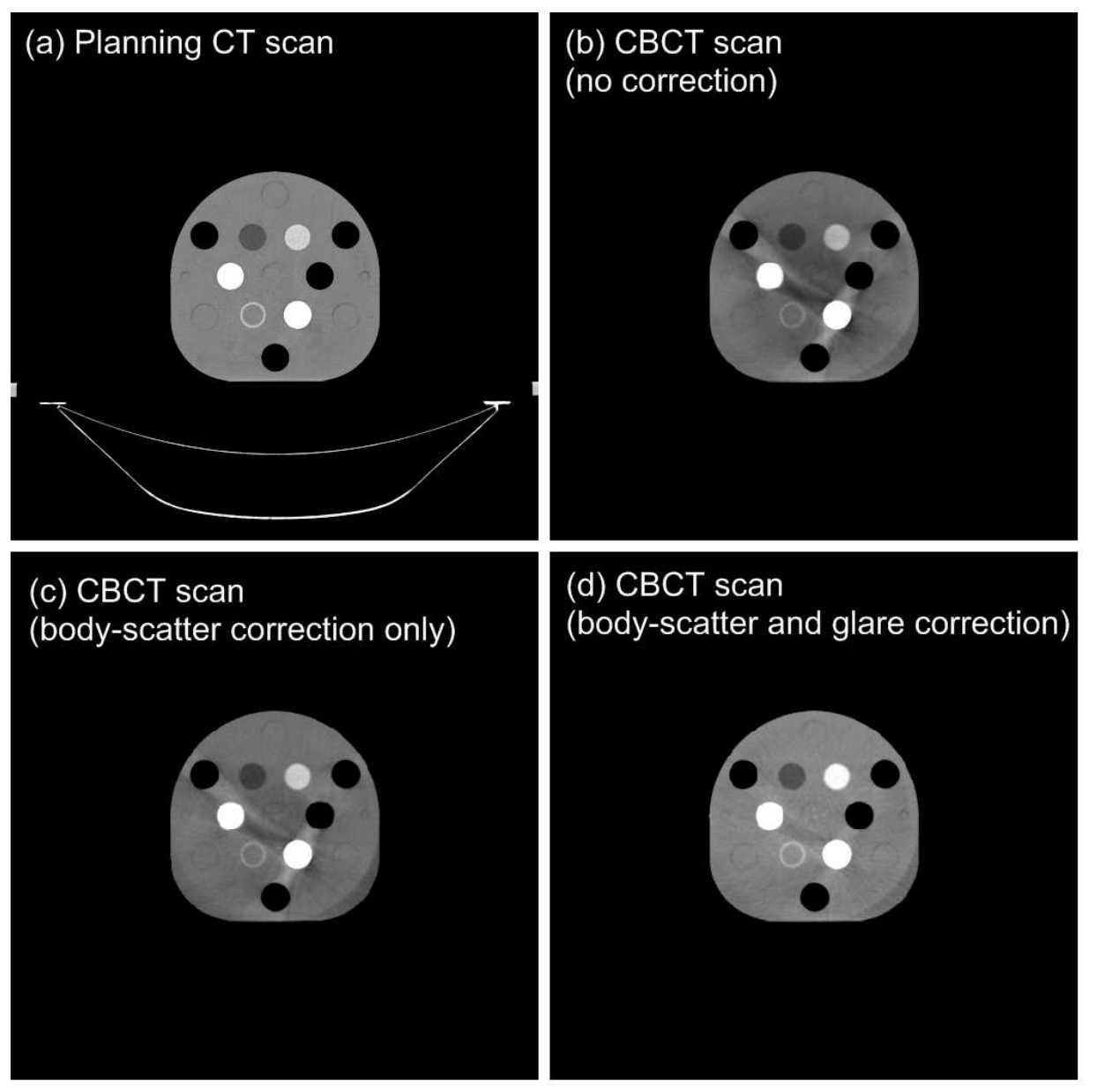

Figure 5. Reconstructions of the HOUNDphan phantom: (a) planning CT scan, (b) CBCT scan (no body-scatter or scatter-glare correction), (c) CBCT scan (body-scatter but no scatter-glare correction) and (d) CBCT scan (body-scatter and scatter-glare correction).

a particular unit of interest, would be preferable. However the results of this work (figures 3 (a), 5 (d) and 6 (a)) suggest that such a generic parameterization may be of use. Moreover the results of table 3 suggest that ignoring the contribution of scatter-glare can impact CT-number fidelity in clinical situations. The tails of the LSF distribution (see figure 3(a)) decline less slowly than generally associated with optical photon spread in scintillators (Kirkby and Sloboda 2005, Freed et al 2009) or secondary-electron diffusion (NIST ESTAR database\|). The glare is therefore likely to be associated with the scatter of x-ray photons in the panel and panel housing, although a contribution from deficiencies in the optical coupling between the scintillator and the thin-film-transistor array cannot be completely discounted. It is somewhat surprising that varying the x-ray tube potential did not have a greater effect on the GSF. The mean energies of the beams varied between about $50 \mathrm{keV}(80 \mathrm{kV})$ and $62 \mathrm{keV}(120$

\| See http://www.nist.gov/physlab/data/star/index.cfm for ESTAR electron ranges. 


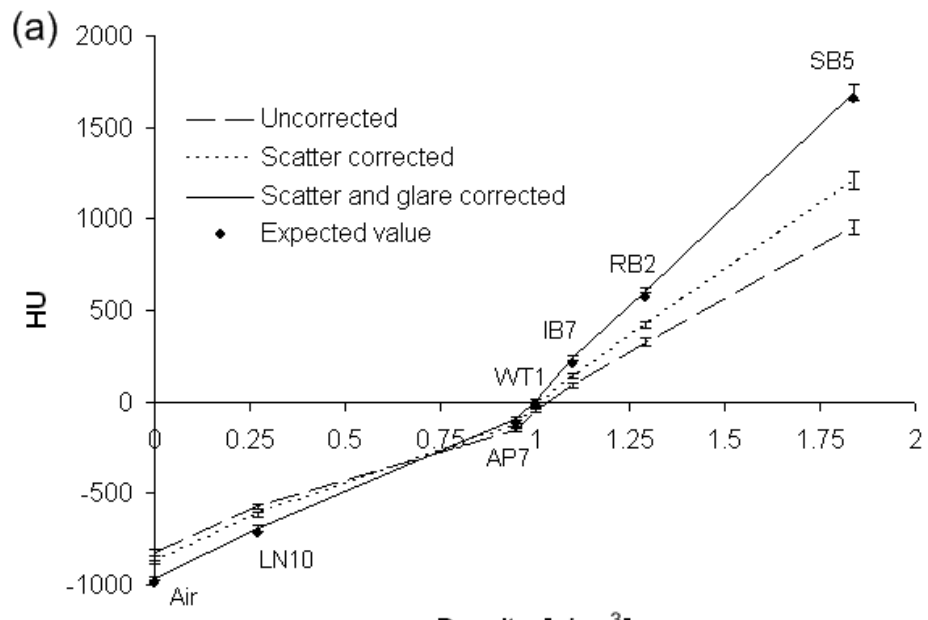

Density $\left[\mathrm{g} / \mathrm{cm}^{3}\right]$

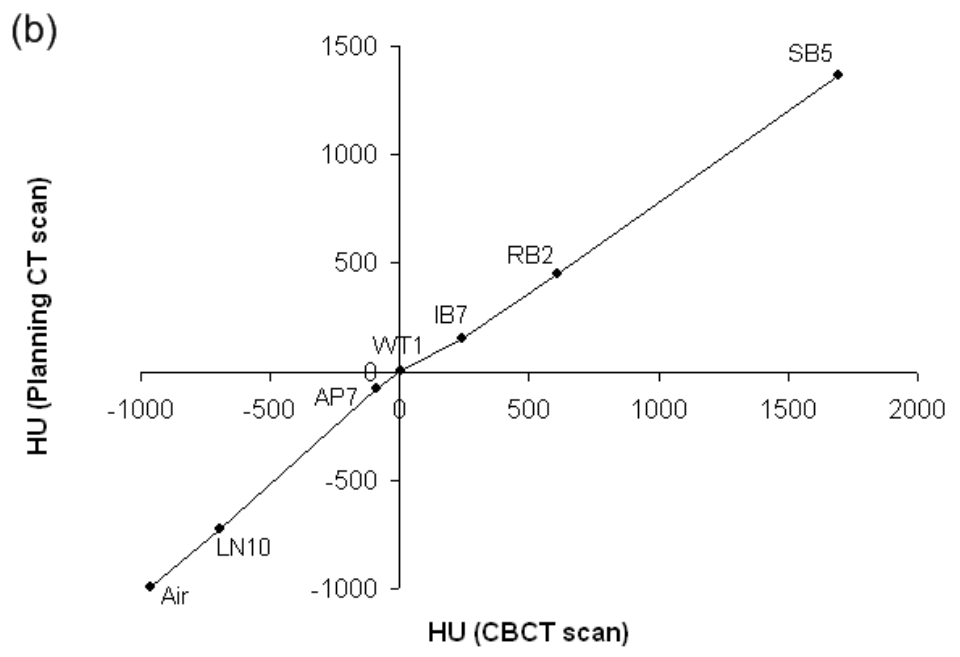

Figure 6. (a) CT-number against density for various materials and (b) crosscalibration curve for the planning CT scan to the CBCT scan.

$\mathrm{kV}$ ) (calculated using the SpekCalc program and HVL data). However, it should be noted that the Compton scattering cross-section is relatively flat in the range 10 to 100 $\mathrm{keV}$, for all materials (NIST XCOM database $\mathbf{)}$ ). In any case, the GSF determination proposed here is straight-forward and requires no specialized or expensive equipment. The deconvolution process, when implemented efficiently, is also not prohibitively timeconsuming. It took approximately $60 \mathrm{~ms}$ to read, deconvolve and write each $512 \times 512$ projection image in the Perkin Elmer HIS format. This was on a Dell Optiplex GX620 PC (Pentium 4 CPU 3.4 GHz, 2GB RAM). The image reconstruction, registration and scatter simulation were also relatively quick. In all cases examined here the entire process took only a few $(<10)$ minutes per data set. The PSF and hence MTF inferred from the edge-spread technique should be interpreted with caution. The aim of this study was not to accurately infer the MTF at higher spatial frequencies. Transmission

ๆ See http://www.nist.gov/physlab/data/xcom/index.cfm for relevant data. 


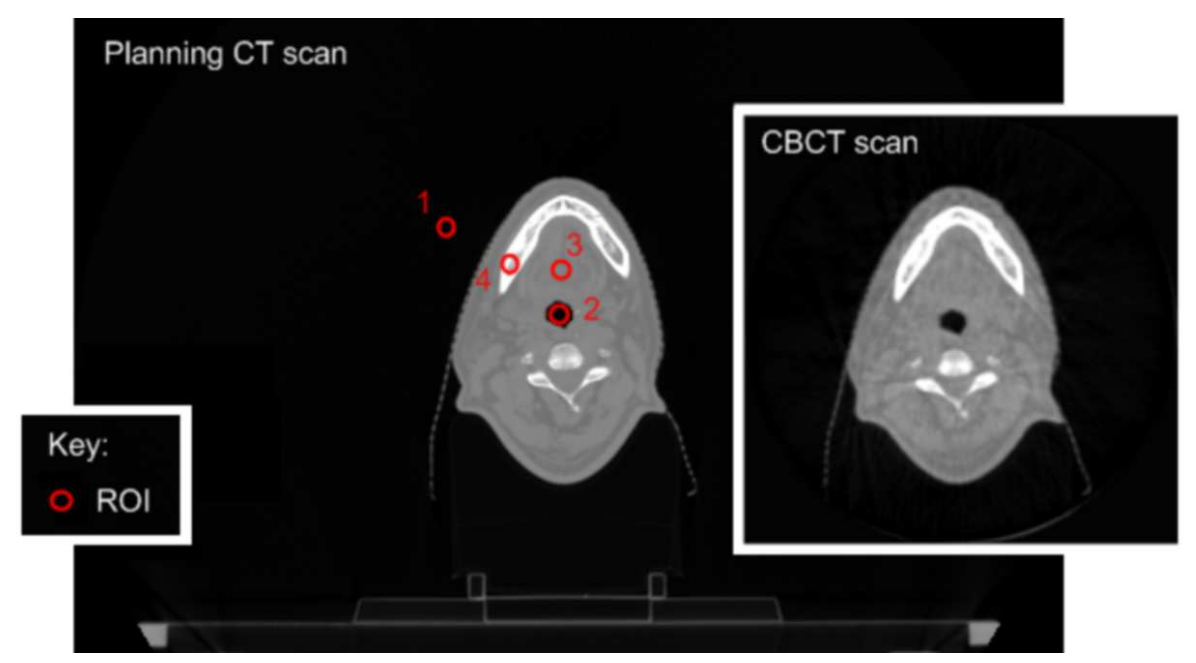

Figure 7. Axial planning CT slice of a head-and-neck cancer patient and the corresponding slice from a treatment-room CBCT scan (inset). Regions-of-interest (ROIs) are shown: 1. air (external), 2. air (hypopharynx), 3. soft-tissue and 4. jaw bone.

of x-rays through the edge of the lead sheeting, imperfections in the machining of the edge, and, any non-perpendicularity of the edge with respect to the pixel rows, will contribute to a broadening of the central PSF peak. The deconvolution with the GSF, however, is only dependent on the parameters $b_{3}$ and $a_{3}$ (describing the tails of the distribution) and therefore any such broadening effects are not critical for the method. We note that our method is similar to that used in other applications. For example, Seibert et al (1984) modelled image-intensifiers and used a deconvolution method to remove veiling-glare. Also, Cooper III et al (2000) applied the deconvolution method to a flat-panel mammography imaging system. Notably, however, our parameterization of the LSF differs and we explore the implications for cone-beam CT reconstructions.

Some empirical approaches to scatter-subtraction in CBCT take scatter-glare into account. We note, for example, the scatter kernel approach of Li et al (2008). The authors' use of an edge-spread technique to estimate the scatter kernels for slabs of material of varying thickness also implicitly includes the contribution of glare. It is unsurprising therefore that their method proved quite successful in correcting CTnumbers. We also note that, recently, Jin et al (2010) have described a variation on the beam-stop array technique (an array of slits) which also provided good results. It is clear therefore that a subtraction of the scatter-glare distribution from experimental projections is a viable alternative to full deconvolution. Simulation (e.g. Monte Carlo) based approaches to the correction of images, however, are quite complimentary to more empirical measurement-based approaches. They can lead to a deeper understanding of the process of image formation and allow the modelling of changes in the imaging process and geometrical setup. They can also, potentially, be of practical use for routine clinical correction. However, whether empirical or simulation methods (or a combination) are 
used for scatter-correction, where scatter-glare has not been accounted for, implicitly or explicitly, CT-number calibration accuracy will be compromised. As we have shown, a reconstruction without compensation for glare will exhibit low-spatial-spatial frequency drop. This explains the remaining CT-number discrepancies in our previous work (Poludniowski et al 2009a) and perhaps the work of others. For example, in Jarry et al (2006), after scatter-correction, Teflon and air still showed reduced contrast compared to predictions (see table 1 of their paper).

The Monte Carlo modeller in CBCT therefore has a number of decisions to make regarding treatment of the PSF/GSF. If the aim of the study is to produce simulated projections (primary plus scatter) that agree, as closely as possible, with those acquired experimentally, then the PSF should probably be modelled. If instead, the aim is scattersubtraction with the object of providing CT reconstructions of the highest CT-number fidelity, then the PSF may be ignored in simulation and the experimental projections corrected for scatter-glare. The practicality of this last approach is shown by the results of this paper (i.e. the results presented in figures 5, 6 and table 3).

\section{Conclusion}

Scatter within a flat-panel detector and housing causes scatter-glare in acquired images.

We have shown that in a commercial CBCT system, the scatter-glare causes low-spatialfrequency drop and has a marked effect on reconstructed CT-number. We suggest a simple method to determine the panel 'GSF' (glare-spread-function) and an effective procedure for deconvolving the glare from the raw projections.

\section{Appendix A. Useful integrals}

The following integrals were used in this work:

$$
\begin{aligned}
& {[3.461(2)]: \int_{0}^{\infty} e^{-y^{2} / 2 b^{2}} d y=\sqrt{\pi b^{2} / 2}} \\
& {[3.365(2)]: \int_{x}^{\infty} \frac{z e^{-z / b} d z}{\sqrt{z^{2}-x^{2}}}=x K_{1}(x / b)} \\
& {[2.271(5)]: \int_{0}^{\infty} \frac{d y}{\left(1+x^{2} / b^{2}+y^{2} / b^{2}\right)^{3 / 2}}=\frac{b}{1+x^{2} / b^{2}}} \\
& {[8.411(1)]: \int_{-\pi}^{\pi} e^{i \omega r \sin \theta} d \theta=2 \pi J_{0}(\omega r)} \\
& {[6.631(4)]: \int_{0}^{\infty} r J_{0}(\omega r) e^{-r^{2} / 2 b^{2}} d r=b^{2} e^{-b^{2} \omega^{2} / 2}} \\
& {[6.623(2)]: \int_{0}^{\infty} r e^{-r / b} J_{0}(\omega r) d r=\frac{b^{2}}{\left(1+b^{2} \omega^{2}\right)^{3 / 2}}} \\
& {[6.554(4)]: \int_{0}^{\infty} r \frac{J_{0}(\omega r)}{\left(1+r^{2} / b^{2}\right)^{3 / 2}} d r=b^{2} e^{-b \omega}}
\end{aligned}
$$

The numbers in brackets before each integral are the reference numbers for appearance in the mathematical tabulations of Gradshteyn and Ryzhik (2000). Note that $J_{0}(\ldots)$ and 
$K_{1}(\ldots)$ are the zeroth order Bessel and first order MacDonald functions, respectively.

\section{Appendix B. Derivation of a glare-spread function}

We present a derivation of the GSF form presented in equation (9). The derivation makes some simplifying assumptions to reach a suggestive form. Let us assume that we have a single thin plane of scattering material located a distance, $b$, from the scintillator layer. The layer is assumed thin enough that only single-scattering contributes. The angles $\phi$ and $\theta$ will represent the azimuthal and zenith scattering angles, respectively. The scatter-distribution function, $f(\theta, \phi)$, is related to the scatter-glare point-spreadfunction, $P S F_{s g}$, by

$$
\int_{0}^{R} r d r \int_{0}^{2 \pi} d \phi P S F_{s g}(r)=\frac{\int_{0}^{\tan ^{-1} \frac{R}{b}} \sin \theta d \theta \int_{0}^{2 \pi} d \phi f(\theta, \phi)}{\int_{0}^{\pi / 2} \sin \theta d \theta \int_{0}^{2 \pi} d \phi f(\theta, \phi)},
$$

where $r$ is the lateral displacement from the position of unscattered x-rays at the scintillator, for an x-ray scattered at the angle, $\theta$. The scatter-distribution will be assumed to be isotropic in direction: i.e. $f(\theta, \phi)=1 / 4 \pi$. We therefore find that:

$$
P S F_{s g}(r)=\frac{1}{2 \pi} \sin \theta \frac{1}{r} \frac{d \theta}{d r}=\frac{1}{2 \pi b^{2}} \frac{1}{\left(1+r^{2} / b^{2}\right)^{3 / 2}} .
$$

If a fraction, $a$, of detected x-rays are scattered before reaching the scintillator, then, the glare-spread-function takes the form,

$$
G S F(r)=(1-a) \frac{\delta(r)}{2 \pi r}+\frac{a}{2 \pi b^{2}} \frac{1}{\left(1+r^{2} / b^{2}\right)^{\frac{3}{2}}},
$$

which is that of (9). Important inferences that therefore can be made about the scatterglare are that:

- The fall-off with $r$ is likely to be sub-exponential;

- A $\left(1+r^{2} / b^{2}\right)^{-\frac{3}{2}}$ function is a good candidate for a fitting-function.

In any empirical fitting to data using the form derived above, however, caution should be taken however in inferring a precise geometrical interpretation of $b$ as the displacement of a single scattering-plane. This is because at least some of the assumptions in the derivation will be weakly violated.

\section{Acknowledgments}

The authors would like to gratefully acknowledge Nigel Wellock of the Tissue Substitute Section of St Bartholomew's Hospital (London, UK) for supplying the tissue-equivalent materials and associated information. We also thank Chris Bunton, from our Institute, for the manufacture of the HOUNDphan phantom and the S2 collimation cassette. The authors would further like to acknowledge Vibeke Hansen for assistance with experimental aspects. This work was partially supported by research grant C46/A2131 from Cancer Research UK. We acknowledge NIHR funding to the NHS Biomedical 
Research Centre. The Image Registration Toolkit was used under Licence from Ixico Ltd. Finally, we thank the referees for their suggestions and corrections to this work.

\section{References}

Barrett HH and Swindell W 1981 Radiological Imaging (Academic Press) pp.60-61.

Boggula R, Lorenz F, Abo-Madyan Y, Lohr F, Wolff D, Boda-Heggemann J, Hesser J, Wenz F and Wertz H 2009 A new strategy for online adaptive prostate radiotherapy based on cone-beam CT Z. Med. Phys. 19(4) 264-76.

Carton AK, Acciavatti R, Kuo J and Maidment AD 2009 The effect of scatter and glare on image quality in contrast-enhanced breast imaging using an a-Si/CsI(TI) full-field flat panel detector Med. Phys. 36(3) 920-8.

Chen L, Shaw CC, Altunbas MC, Lai CJ and Liu X 2008 Spatial resolution properties in cone beam CT: a simulation study Med. Phys. 35(2) 724-34.

Cooper VN III, Boone JM, Seibert JA and Pellot-Barakat CJ 2000 An edge spread technique for measurement of the scatter-to-primary ratio in mammography Med. Phys. 27(5) 845-53.

Den RB, Doemer A, Kubicek G, Bednarz G, Galvin JM, Keane WM, Xiao Y and Machtay M 2009 Daily image guidance with cone-beam computed tomography for head-and-neck cancer intensity-modulated radiotherapy: a prospective study Int J Radiat Oncol. Biol. Phys. 2010 76(5) 1353-9.

Feldkamp LA, Davis LC and Kress JW 1984 Practical cone-beam algorithm JOSA A, 1(6) 612-619.

Freed M, Miller S, Tang K and Badano A 2009 Experimental validation of Monte Carlo (MANTIS) simulated x-ray response of columnar CsI scintillator screens Med. Phys. 2009 36(11) 4944-56.

Friedman SN and Cunningham IA 2008 Normalization of the modulation transfer function: the openfield approach Med. Phys. 35(10) 4443-9.

Gradshteyn IS and Ryzhik IM 2000 Table of Integrals, Series, and Products 6th edition (Academic Press).

Illers H, Buhr E, Gnther-Kohfahl S and Neitzel U 2005 Measurement of the modulation transfer function of digital X-ray detectors with an opaque edge-test device Radiat. Prot. Dosimetry. 114(1-3) 214-9.

Jarry G, Graham SA, Moseley DJ, Jaffray DJ, Siewerdsen JH and Verhaegen F 2006 Characterization of scattered radiation in $k V$ CBCT images using Monte Carlo simulations. Med. Phys. 33(11) 4320-9.

Jin J-Y, Ren L, Liu Q, Kim J, Wen N, Movsas B and Chetty IJ 2010 Methods to fully generate improved $C B C T$ images from partially-blocked projection data Proc. of the 16th International Conference on the Use of Computers in Radiation Therapy (ICCR) (Het Nederlands Kanker Instituut- Antoni van Leeuwenhoek Ziekenhuis, Amsterdam).

Kak A C and Slaney M 2001 Principles of Computerized Tomographic Imaging Chapter 3 Algorithms for Reconstruction with Nondiffracting Sources (SIAM) (Philadelphia, USA) p 49-112.

Kirkby C and Sloboda R 2005 Comprehensive Monte Carlo calculation of the point spread function for a commercial a-Si EPID Med. Phys. 2005 32(4) 1115-27.

Lebedev NN 1972 Special Functions and Their Applications Chapter 5 Cylinder Functions: Theory (Dover Publications, Inc.) (New York, USA) p 108-109.

Li H, Mohan R and Zhu XR 2008 Scatter kernel estimation with an edge-spread function method for cone-beam computed tomography imaging Phys. Med. Biol. 53(23) 6729-48.

Lu W, Chen M, Chen Q, Ruchala K and Olivera G 2008 Adaptive fractionation therapy: I. Basic concept and strategy Phys. Med. Biol. 53 5495-511.

Mail N, Moseley DJ, Siewerdsen JH and Jaffray DA 2008 An empirical method for lag correction in cone-beam CT Med. Phys. 2008 35(11) 5187-96.

Marchant TE, Moore CJ, Rowbottom CG, MacKay RI and Williams PC 2008 Shading correction algorithm for improvement of cone-beam CT images in radiotherapy Phys. Med. Biol. 53(20) 571933.

Ploeger LS, Sonke JJ van Herk M 2010 Characterization of ghosting in cone beam CT Proc. of the 16th 
International Conference on the Use of Computers in Radiation Therapy (ICCR) (Het Nederlands Kanker Instituut- Antoni van Leeuwenhoek Ziekenhuis, Amsterdam).

Richter A, Hu Q, Steglich D, Baier K, Wilbert J, Guckenberger M and Flentje M 2008 Investigation of the usability of conebeam CT data sets for dose calculation Radiat. Oncol. 16(3) 42.

Poludniowski GG, Evans PM and Webb S 2009a An efficient Monte Carlo-based algorithm for scatter correction in keV cone-beam CT Phys. Med. Biol. 54(12) 3847-3864.

Poludniowski G, Landry G, DeBlois F, Evans PM and Verhaegen F 2009b SpekCalc: a program to calculate photon spectra from tungsten anode x-ray tubes Phys. Med. Biol. bf 54(19) N433-8.

Poludniowski GG 2007 Calculation of $x$-ray spectra emerging from an x-ray tube. Part II. X-ray production and filtration in x-ray targets Med. Phys. 34(6) 2175-76.

Roberts DA, Hansen V N, Niven A C, Thompson M G, Seco J and Evans P M 2008 A low Z linac and flat panel imager: comparison with the conventional imaging approach Phys. Med. Biol. 53(22) 6305-19.

Seibert JA, Nalcioglu O and Roeck WW 1984 Characterization of the veiling glare PSF in x-ray image intensified fluoroscopy Med. Phys. 11(2) 172-9.

Siewerdsen JH and Jaffray DA 2001 Cone-beam computed tomography with a flat-panel imager: magnitude and effects of $x$-ray scatter Med. Phys. 28(2) 220-31.

Siewerdsen JH and Jaffray DA 1999 A ghost story: spatio-temporal response characteristics of an indirect-detection flat-panel imager Med. Phys. 26(8) 1624-41.

Studholme C, Hill DLG, Hawkes DJ 1999 An Overlap Invariant Entropy Measure of 3D Medical Image Alignment, Pattern Recognition 32(1) 71-86.

Webb S 2008 Adapting IMRT delivery fraction-by-fraction to cater for variable intrafraction motion Phys. Med. Biol. 53 1-21. 